\title{
Baryonic Q-balls as Dark Matter
}

\author{
Alexander Kusenko \\ Department of Physics and Astronomy, UCLA, Los Angeles, CA 90095-1547 \\ RBRC, Brookhaven National Laboratory, Upton, New York 11973
}

\begin{abstract}
Supersymmetric extensions of the Standard Model predict the existence of Q-balls, some of which can be entirely stable. Affleck-Dine baryogenesis can result in a copious production of stable baryonic Q-balls, which can presently exist as a form of dark matter.
\end{abstract}

\section{Q-balls from Supersymmetry}

In a class of theories with interacting scalar fields $\phi$ that carry some conserved global charge, the ground state is a Q-ball [1,2], a lump of coherent scalar condensate that can be described semiclassically as a non-topological soliton of the form

$$
\phi(x, t)=e^{i \omega t} \bar{\phi}(x) .
$$

Q-balls exist whenever the scalar potential satisfies certain conditions that were first derived for a single scalar degree of freedom [1] with some abelian global charge and were later generalized to a theory of many scalar fields with different charges [3]. Non-abelian global symmetries [4] and abelian local symmetries [5] can also yield Q-balls.

For a simple example, let us consider a field theory with a scalar potential $U(\varphi)$ that has a global minimum $U(0)=0$ at $\varphi=0$. Let $U(\varphi)$ have an unbroken global[ $\mathrm{U}(1)$ symmetry at the origin, $\varphi=0$. And let the scalar field $\varphi$ have a unit charge with respect to this $\mathrm{U}(1)$.

The charge of some field configuration $\varphi(x, t)$ is

$$
Q=\frac{1}{2 i} \int \varphi^{*} \stackrel{\leftrightarrow}{\partial}_{t} \varphi d^{3} x
$$

Since a trivial configuration $\varphi(x) \equiv 0$ has zero charge, the solution that minimizes the energy,

$$
E=\int d^{3} x\left[\frac{1}{2}|\dot{\varphi}|^{2}+\frac{1}{2}|\nabla \varphi|^{2}+U(\varphi)\right]
$$

and has a given charge $Q>0$, must differ from zero in some (finite) domain. This is a Q-ball. It is a time-dependent solution, more precisely it has

1 Q-balls associated with a local symmetry have been constructed [5]. An important qualitative difference is that, in the case of a local symmetry, there is an upper limit on the charge of a stable Q-ball. 
a time-dependent phase. However, all physical quantities are time-independent. Of course, we have not proven that such a "lump" is finite, or that it has a lesser energy than the collection of free particles with the same charge; neither is true for a general potential. A finite-size Q-ball is a minimum of energy and is stable with respect to decay into free $\varphi$-particles if

$$
U(\varphi) / \varphi^{2}=\min , \text { for } \varphi=\varphi_{0}>0 .
$$

One can show that the equations of motion for a $\mathrm{Q}$-ball in $3+1$ dimensions are equivalent to those for the bounce associated with tunneling in 3 Euclidean dimensions in an effective potential $\hat{U}_{\omega}(\varphi)=U(\varphi)-(1 / 2) \omega^{2} \varphi^{2}$, where $\omega$ is such that it extremizes 7

$$
\mathcal{E}_{\omega}=S_{3}(\omega)+\omega Q .
$$

Here $S_{3}(\omega)$ is the three-dimensional Euclidean action of the bounce in the potential $\hat{U}_{\omega}(\varphi)$. The Q-ball solution has the form (1), where $\bar{\varphi}(x)$ is the bounce.

The analogy with tunneling clarifies the meaning of condition (14), which simply requires that there exist a value of $\omega$, for which $\hat{U}_{\omega}(\varphi)$ is negative for some value of $\varphi=\varphi_{0} \neq 0$ separated from the false vacuum by a barrier. This condition ensures the existence of a bounce. (Clearly, the bounce does not exist if $\hat{U}_{\omega}(\varphi) \geq 0$ for all $\varphi$ because there is nowhere to tunnel.)

In the true vacuum, there is a minimal value $\omega_{0}$, so that only for $\omega>\omega_{0}$, $\hat{U}_{\omega}(\varphi)$ is somewhere negative. If one considers a Q-ball in a metastable false vacuum, then $\omega_{0}=0$. The mass of the $\varphi$ particle is the upper bound on $\omega$ in either case. Large values of $\omega$ correspond to small charges [7]. As $Q \rightarrow \infty$, $\omega \rightarrow \omega_{0}$. In this case, the effective potential $\hat{U}_{\omega}(\varphi)$ has two nearly-degenerate minima; and one can apply the thin-wall approximation to calculate the Q-ball energy [1]. For smaller charges, the thin-wall approximation breaks down, and one has to resort to other methods [7].

The above discussion can be generalized to the case of several fields, $\varphi_{k}$, with different charges, $q_{k}[3]$. Then the Q-ball is a solution of the form

$$
\varphi_{k}(x, t)=e^{i q_{k} \omega t} \varphi_{k}(x),
$$

where $\varphi(x)$ is again a three-dimensional bounce associated with tunneling in the potential

$$
\hat{U}_{\omega}(\varphi)=U(\varphi)-\frac{1}{2} \omega^{2} \sum_{k} q_{k}^{2}\left|\varphi_{k}\right|^{2} .
$$

As before, the value of $\omega$ is found by minimizing $\mathcal{E}_{\omega}$ in equation (5). The bounce, and, therefore, the Q-ball, exists if

$$
\begin{aligned}
& \mu^{2}=2 U(\varphi) /\left(\sum_{k} q_{k} \varphi_{k, 0}^{2}\right)=\min \\
& \quad \text { for }\left|\boldsymbol{\varphi}_{0}\right|^{2}>0
\end{aligned}
$$

The soliton mass can be calculated by extremizing $\mathcal{E}_{\omega}$ in equation (5). If $\left|\boldsymbol{\varphi}_{0}\right|^{2}$ defined by equation ( 8 is finite, then the mass of a soliton $M(Q)$ is proportional 
to the first power of $Q$ :

$$
M(Q)=\tilde{\mu} Q, \quad \text { if }\left|\varphi_{0}\right|^{2} \neq \infty .
$$

In particular, if $Q \rightarrow \infty, \tilde{\mu} \rightarrow \mu$ (thin-wall limit) [1,2]. For smaller values of $Q$, $\tilde{\mu}$ was computed in [f]. In any case, $\tilde{\mu}$ is less than the mass of the $\phi$ particle by definition (8).

However, if the scalar potential grows slower than the second power of $\phi$, then $\left|\boldsymbol{\varphi}_{0}\right|^{2}=\infty$, and the Q-ball never reaches the thin-wall regime, even if $\mathrm{Q}$ is large. The value of $\phi$ inside the soliton extends as far as the gradient terms allow, and the mass of a Q-ball is proportional to $Q^{p}, p<1$. In particular, if the scalar potential has a flat plateau $U(\phi) \sim m$ at large $\phi$, then the mass of a Q-ball is 11]

$$
M(Q) \sim m Q^{3 / 4}
$$

This is the case for the stable baryonic Q-balls in the MSSM discussed below.

It turns out that all phenomenologically viable supersymmetric extensions of the Standard Model predict the existence of non-topological solitons [3] associated with the conservation of baryon and lepton number. If the physics beyond the standard model reveals some additional global symmetries, this will further enrich the spectrum of Q-balls [6]. The MSSM admits a large number of different Q-balls, characterized by (i) the quantum numbers of the fields that form a spatially-inhomogeneous ground state and (ii) the net global charge of this state.

First, there is a class of Q-balls associated with the tri-linear interactions that are inevitably present in the MSSM [3]. The masses of such Q-balls grow linearly with their global charge, which can be an arbitrary integer number [7]. Baryonic and leptonic Q-balls of this variety are, in general, unstable with respect to their decay into fermions. However, they could form in the early universe through the accretion of global charge [8,9] or, possibly, in a first-order phase transition [10].

The second class [11] of solitons comprises the Q-balls whose VEVs are aligned with some flat directions of the MSSM. The scalar field inside such a Q-ball is a gauge-singlet [12] combination of squarks and sleptons with a nonzero baryon or lepton number. The potential along a flat direction is lifted by some soft supersymmetry-breaking terms that originate in a "hidden sector" of the theory at some scale $\Lambda_{S}$ and are communicated to the observable sector by some interaction with a coupling $g$, so that $g \Lambda \sim 100 \mathrm{GeV}$. Depending on the strength of the mediating interaction, the scale $\Lambda_{S}$ can be as low as a few TeV (as in the case of gauge-mediated SUSY breaking), or it can be some intermediate scale if the mediating interaction is weaker (for instance, $g \sim \Lambda_{S} / m_{\text {Planck }}$ and $\Lambda_{S} \sim 10^{10} \mathrm{GeV}$ in the case of gravity-mediated SUSY breaking). For the lack of a definitive scenario, one can regard $\Lambda_{S}$ as a free parameter. Below $\Lambda_{S}$ the mass terms are generated for all the scalar degrees of freedom, including those that parameterize the flat direction. At the energy scales larger than $\Lambda_{S}$, the mass terms turn off and the potential is "flat" up to some logarithmic corrections. If the Q-ball VEV extends beyond $\Lambda_{S}$, the mass of a soliton [11, 13] is no longer proportional to its global charge $Q$, but rather to $Q^{3 / 4}$. A hybrid of the two types is yet another possibility 14 . 
This allows for the existence of some entirely stable Q-balls with a large baryon number $B$ (B-balls). Indeed, if the mass of a B-ball is $M_{B} \sim(1 \mathrm{TeV}) \times$

$B^{3 / 4}$, then the energy per baryon number $\left(M_{B} / B\right) \sim(1 \mathrm{TeV}) \times B^{-1 / 4}$ is less than $1 \mathrm{GeV}$ for $B>10^{12}$. Such large B-balls cannot dissociate into protons and neutrons and are entirely stable thanks to the conservation of energy and the baryon number. If they were produced in the early universe, they would exist at present as a form of dark matter 13].

\section{Fragmentation of Affleck-Dine Condensate into Q-balls}

Several mechanisms could lead to formation of B-balls and L-balls in the early universe. First, they can be produced in the course of a phase transition [10]. Second, thermal fluctuations of a baryonic and leptonic charge can, under some conditions, form a Q-ball. Finally, a process of a gradual charge accretion, similar to nucleosynthesis, can take place [8,9,15]. However, it seems that the only process that can lead to a copious production of very large, and, hence, stable, B-balls, is fragmentation of the Affleck-Dine condensate [13].

At the end of inflation, the scalar fields of the MSSM develop some large expectation values along the flat directions, some of which have a non-zero baryon number [16]. Initially, the scalar condensate has the form given in eq. (11) with $\bar{\phi}(x)=$ const over the length scales greater than a horizon size. One can think of it as a universe filled with Q-matter. The relaxation of this condensate to the potential minimum is the basis of the Affleck-Dine (AD) scenario for baryogenesis.

It was often assumed that the condensate remains spatially homogeneous from the time of formation until its decay into the matter baryons. This assumption is, in general, incorrect. In fact, the initially homogeneous condensate can become unstable 113 and break up into Q-balls whose size is determined by the potential and the rate of expansion of the Universe. B-balls with $12<\log _{10} B<30$ can form naturally from the breakdown of the AD condensate. These are entirely stable if the flat direction is "sufficiently flat", that is if the potential grows slower than $\phi^{2}$ on the scales or the order of $\bar{\phi}(0)$. The evolution of the primordial condensate can be summarized as follows: 


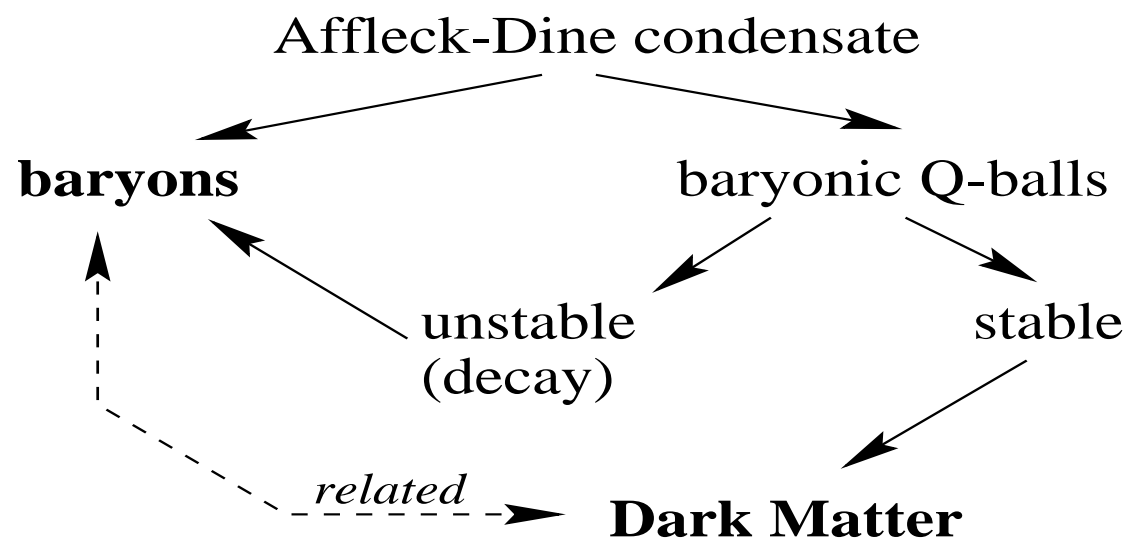

This process has been analyzed analytically [13,22] in the linear approximation. Recently, some impressive numerical simulations of Q-ball formation have been performed [23]; they confirm that the fragmentation of the condensate into Q-balls occurs in some Affleck-Dine models. The global charges of Q-balls that form this way are model dependent. The subsequent collisions [13,24] can further modify the distribution of soliton sizes.

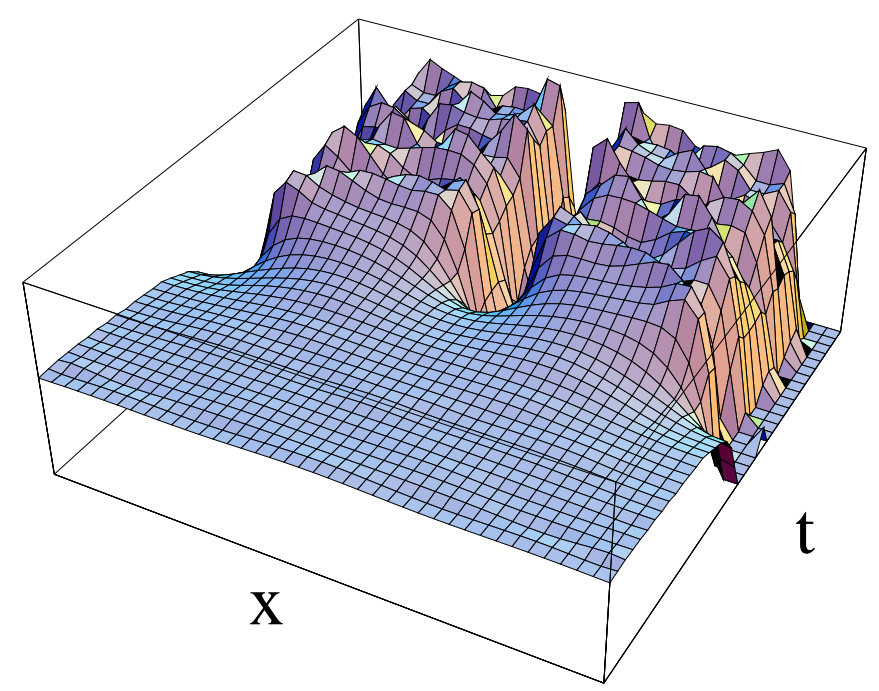

Fig. 1. The charge density per comoving volume in $(1+1)$ dimensions for a sample potential analyzed numerically during the fragmentation of the condensate into Q-balls.

In supersymmetric extensions of the Standard Model, Q-ball formation occurs along flat directions of a certain type, which appear to be generic in the MSSM [25]. 


\section{SUSY Q-balls as Dark Matter}

Conceivably, the cold dark matter in the Universe can be made up entirely of SUSY Q-balls. Since the baryonic matter and the dark matter share the same origin in this scenario, their contributions to the mass density of the Universe are related. Most of dark-matter scenarios offer no explanation as to why the observations find $\Omega_{D A R K} \sim \Omega_{B}$ within an order of magnitude. This fact is extremely difficult to explain in models that invoke a dark-matter candidate whose present-day abundance is determined by the process of freeze-out, independent of baryogenesis. If one doesn't want to accept this equality as fortuitous, one is forced to hypothesize some ad hoc symmetries [26] that could relate the two quantities. In the MSSM with AD baryogenesis, the amounts of dark-matter Q-balls and the ordinary matter baryons are related 13; one predicts 17] $\Omega_{D A R K}=\Omega_{B}$ for B-balls with $B \sim 10^{26}$. However, the size of Q-balls depends on the supersymmetry breaking terms that lift the flat direction. The required size is in the middle of the range of Q-ball sizes that can form in the Affleck-Dine scenario [13,22,23]. Diffusion effects may force the Q-balls sizes to be somewhat smaller, $B \sim 10^{22}-10^{24}$, if they are to be CDM and to generate the baryon asymmetry of the universe through partial evaporation [18].

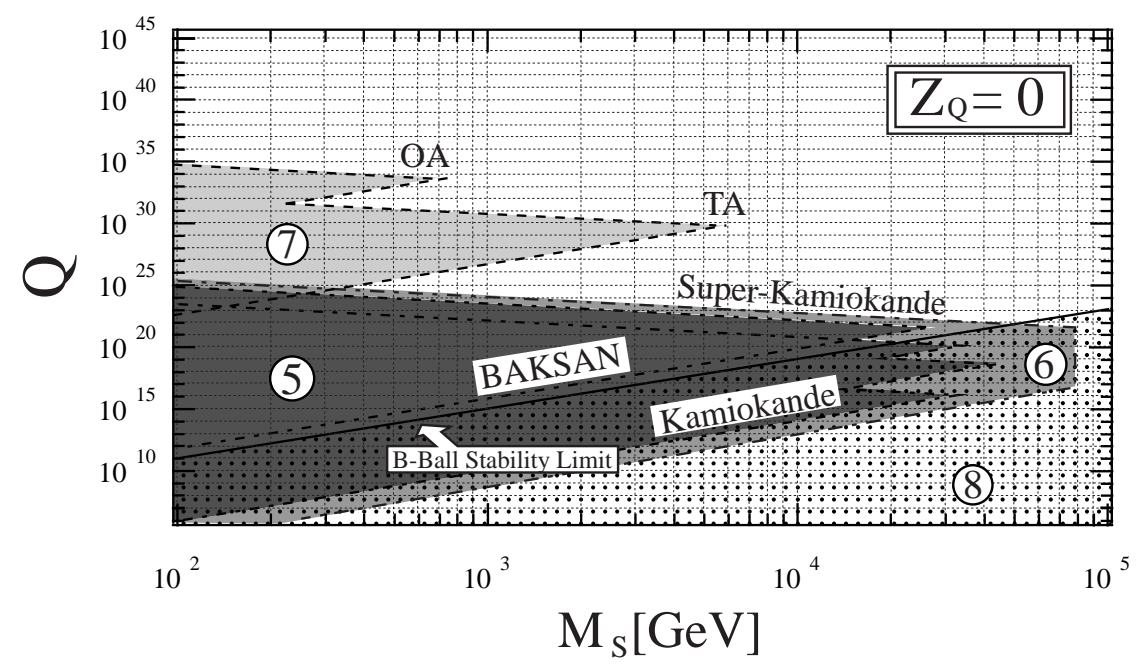

Fig. 2. The resent limits on the baryon numbers of electrically neural dark-matter Q-balls from a paper by J. Arafune et al. 28.

The value $B \sim 10^{26}$ is well within the present experimental limits on the baryon number of an average relic B-ball, under the assumption that all or most of cold dark matter is made up of Q-balls. On their passage through matter, the electrically neutral baryonic SUSY Q-balls can cause a proton decay, while the electrically charged B-balls produce massive ionization. Although the condensate 
inside a Q-ball is electrically neutral [12], it may pick up some electric charge through its interaction with matter 19]. Regardless of its ability to retain electric charge, the Q-ball would produce a straight track in a detector and would release the energy of, roughly, $10 \mathrm{GeV} / \mathrm{mm}$. The present limits [19,27,28 constrain the baryon number of a relic dark-matter B-ball to be greater than $10^{22}$. Future experiments are expected to improve these limits. It would take a detector with the area of several square kilometers to cover the entire interesting range $B \sim$ $10^{22} \ldots 10^{30}$.

\section{Star Wreck: the Q-ball Invasion}

In non-supersymmetric theories, nuclear matter of neutron stars is the lowestenergy state with a given baryon number ${ }^{2}$. In supersymmetric theories, however, a Q-ball with baryon number $10^{57}$ can be lighter than a neutron star. I am going to describe a process that can transform a neutron star into a very large B-ball. The time scale involved is naturally of the order of billion years.

Dark-matter superballs pass through the ordinary stars and planets with a negligible change in their velocity. However, both SECS and SENS stop in the neutron stars and accumulate there [20]. As soon as the first Q-ball is captured by a neutron star, it sinks to the center and begins to absorb the baryons into the condensate. High baryon density inside a neutron star makes this absorption very efficient, and the B-ball grows at the rate that increases with time due to the gradual increase in the surface area. After some time, the additional dark-matter Q-balls that fall onto the neutron star make only a negligible contribution to the growth of the central Q-ball [20]. So, the fate of the neutron star is sealed when it captures the first superball.

According to the discussion in section 3, the energy per unit baryon number inside the relic B-ball is less than that in nuclear matter. Therefore, the absorption process is accompanied by the emission of heat carried away by neutrinos and photons. We estimate that this heating is too weak to lead to any observable consequences. However, the absorption of nuclear matter by a baryonic Q-ball causes a gradual decrease in the mass of the neutron star.

Neutron stars are stable in some range of masses. In particular, there is a minimal mass (about 0.18 solar mass), below which the force of gravity is not strong enough to prevent the neutrons from decaying into protons and electrons. While the star is being consumed by a superball, its mass gradually decreases, reaching the critical value eventually. At that point, a mini-supernova explosion occurs [30], which can be observable. Perhaps, the observed gamma-ray bursts may originate from an event of this type. A small geometrical size of a neutron star and a large energy release may help reconcile the brightness of the gammaray bursts with their short duration.

\footnotetext{
${ }^{2}$ I remind the reader that black holes do not have a well-defined baryon number.
} 
Depending on the MSSM parameters, the lifetime of a neutron star $t_{s}$ can range from 0.01 Gyr to more than 10 Gyr [20]:

$$
t_{s} \sim \frac{1}{\beta} \times\left(\frac{m}{200 \mathrm{GeV}}\right)^{5} \mathrm{Gyr},
$$

where $\beta$ is some model-dependent quantity expected to be of order one 20]. The ages of pulsars set the limit $t_{s}>0.1 \mathrm{Gyr}$.

It is interesting to note that $t_{s}$ depends on the fifth power of the mass parameter $m$ associated with supersymmetry breaking. If the mini-supernovae are observed (or if the connection with gamma-ray bursts is firmly established), one can set strict constraints on the supersymmetry breaking sector from the rate of neutron star explosions.

The naturally long time scale is intriguing.

\section{B-ball Baryogenesis}

An interesting scenario that relates the amounts of baryonic and dark matter in the Universe, and in which the dark-matter particles are produced from the decay of unstable B-balls was proposed by Enqvist and McDonald 22].

\section{Phase Transitions Precipitated by Solitosynthesis}

In the false vacuum, a rapid growth of non-topological solitons [8] can precipitate an otherwise impossible or slow phase transition [9].

Let us suppose the system is in a metastable false vacuum that preserves some $\mathrm{U}(1)$ symmetry. The potential energy in the Q-ball interior is positive in the case of a true vacuum, but negative if the system is in the metastable false vacuum. In either case, it grows as the third power of the Q-ball radius $R$. The positive contribution of the time derivative to the soliton mass can be written as $Q^{2} / \int \bar{\phi}^{2}(x) d^{3} x \propto R^{-3}$, and the gradient surface energy scales as $R^{2}$. In the true vacuum, all three contributions are positive and the Q-ball is the absolute minimum of energy (Fig. 3). However, in the false vacuum, the potential energy inside the Q-ball is negative and goes as $\propto-R^{3}$. As shown in Fig. 3, for small charge $Q$, there are two stationary points, the minimum and the maximum. The former corresponds to a Q-ball (which is, roughly, as stable as the false vacuum is), while the latter is a critical bubble of the true vacuum with a non-zero charge.

There is a critical value of charge $Q=Q_{c}$, for which the only stationary point is unstable. If formed, such an unstable bubble will expand.

If the Q-ball charge increases gradually, it eventually reaches the critical value. At that point Q-ball expands and converts space into a true-vacuum phase. In the case of tunneling, the critical bubble is formed through coincidental coalescence of random quanta into an extended coherent object. This is a smallprobability event. If, however, a Q-ball grows through charge accretion, it reaches the critical size with probability one, as long as the conditions for growth [9] are satisfied. The phase transition can proceed at a much faster rate than it would by tunneling. 


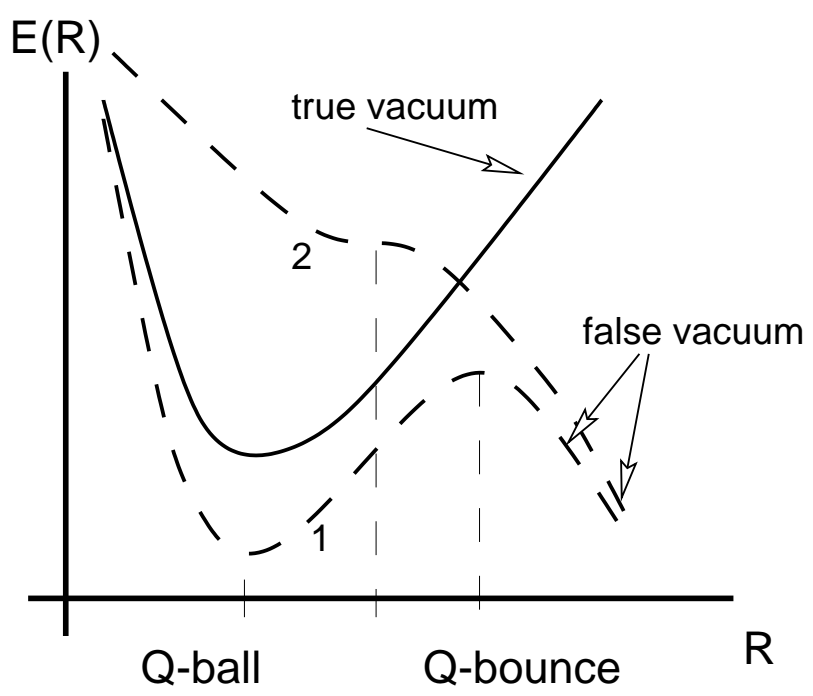

Fig. 3. Energy (mass) of a soliton as a function of its size. In the true vacuum, Q-ball is the global minimum of energy (solid curve). In the false vacuum, if the charge is less than some critical value, there are two solutions: a "stable" Q-ball, and an unstable "Q-bounce" (dashed curve 1). In the case of a critical charge (curve 2), there is only one solution, which is unstable.

\section{Conclusion}

Supersymmetric models of physics beyond the weak scale offer two plausible candidates for cold dark matter. One is the lightest supersymmetric particle, which is stable because of R-parity. Another one is a stable non-topological soliton, or Q-ball, carrying some baryonic charge.

SUSY Q-balls make an appealing dark-matter candidate because their formation is a natural outcome of Affleck-Dine baryogenesis and requires no unusual assumptions.

In addition, formation and decay of unstable Q-balls can have a dramatic effect on baryogenesis, dark matter, and the cosmic microwave background. Production of unstable Q-balls in the false vacuum can cause an unusually fast first-order phase transition.

My work was supported in part by the US Department of Energy grant DEFG03-91ER40662, Task C, and by a UCLA Council on Research faculty grant.

\section{References}

1. G. Rosen: J. Math. Phys. 9, 996 (1968) ibid. 9, 999 (1968); R. Friedberg, T. D. Lee, A. Sirlin: Phys. Rev. D 13, 2739 (1976); S. Coleman: Nucl. Phys. B 262, 263 (1985)

2. T. D. Lee and Y. Pang, Phys. Rept. 221, 251 (1992)

3. A. Kusenko: Phys. Lett. B 405, 108 (1997) 
4. A. M. Safian, S. Coleman, M. Axenides: Nucl. Phys. B 297, 498 (1988); A. M. Safian: Nucl. Phys. B 304, 392 (1988); M. Axenides: Int. J. Mod. Phys. A 7, 7169 (1992); M. Axenides, E. Floratos, A. Kehagias: Phys. Lett. B 444, 190 (1998)

5. K. Lee, J. A. Stein-Schabes, R. Watkins, L. M. Widrow: Phys. Rev. D 39, 1665 (1989); T. Shiromizu, T. Uesugi, M. Aoki: Phys. Rev. D 59, 125010 (1999)

6. D. A. Demir: Phys. Lett. B 450, 215 (1999); D. A. Demir: hep-ph/0006344

7. A. Kusenko: Phys. Lett. B 404, 285 (1997), hep-th/9704073

8. K. Griest, E. W. Kolb: Phys. Rev. D 40, 3231 (1989); J. A. Frieman, A. V. Olinto, M. Gleiser, C. Alcock: Phys. Rev. D 40, 3241 (1989)

9. A. Kusenko: Phys. Lett. B 406, 26 (1997)

10. J. A. Frieman, G. B. Gelmini, M. Gleiser, E. W. Kolb: Phys. Rev. Lett. 60, 2101 (1988); K. Griest, E. W. Kolb, A. Maassarotti, Phys. Rev. D 40, 3529 (1989); J. Ellis, J. Hagelin, D. V. Nanopoulos, K. Tamvakis: Phys. Lett. B 125, 275 (1983)

11. G. Dvali, A. Kusenko, M. Shaposhnikov: Phys. Lett. B 417, 99 (1998)

12. A. Kusenko, M. Shaposhnikov, P. G. Tinyakov: Pisma Zh. Eksp. Teor. Fiz. 67, 229 (1998)

13. A. Kusenko, M. Shaposhnikov: Phys. Lett. B 418, 46 (1998)

14. S. Kasuya and M. Kawasaki, hep-ph/0006128.

15. S. Khlebnikov, I. Tkachev: Phys. Rev. D61, 083517 (2000)

16. I. Affleck, M. Dine: Nucl. Phys. B 249, 361 (1985); M. Dine, L. Randall, S. Thomas: Phys. Rev. Lett. 75, 398 (1995); Nucl. Phys. B 458, 291 (1996); A. Anisimov and M. Dine: hep-ph/0008058

17. M. Laine, M. Shaposhnikov: Nucl. Phys. B 532, 376 (1998)

18. R. Banerjee and K. Jedamzik, Phys. Lett. B484, 278 (2000)

19. A. Kusenko, V. Kuzmin, M. Shaposhnikov, P. G. Tinyakov: Phys. Rev. Lett. 80, $3185(1998)$

20. A. Kusenko, M. Shaposhnikov, P. G. Tinyakov, I. I. Tkachev: Phys. Lett. B 423, $104(1998)$

21. M. Boz, D. A. Demir and N. K. Pak, Mod. Phys. Lett. A15, 517 (2000)

22. K. Enqvist, J. McDonald: Phys. Lett. B 425, 309 (1998); Nucl. Phys. B 538, 321 (1999); Phys. Rev. Lett. 81, 3071 (1998); Phys. Lett. B 440, 59 (1998); Phys. Rev. Lett. 83, 2510 (1999)

23. S. Kasuya, M. Kawasaki: Phys. Rev. D 61, 041301 (2000); S. Kasuya and M. Kawasaki: Phys. Rev. D62, 023512 (2000)

24. M. Axenides, S. Komineas, L. Perivolaropoulos, M. Floratos: Phys. Rev. D61, 085006 (2000); R. Battye and P. Sutcliffe: hep-th/0003252; T. Multamaki and I. Vilja, Phys. Lett. B482, 161 (2000); T. Multamaki and I. Vilja: Phys. Lett. B484, 283 (2000)

25. K. Enqvist, A. Jokinen and J. McDonald, Phys. Lett. B483, 191 (2000)

26. D. B. Kaplan: Phys. Rev. Lett. 68, 741 (1992)

27. I. A. Belolaptikov et al.: astro-ph/9802223 M. Ambrosio et al. [MACRO Collaboration]: Eur. Phys. J. C13, 453 (2000) B. C. Choudhary [MACRO Collaboration]: hep-ex/9905023 G. Giacomelli, L. Patrizii: DFUB-98-30, hep-ex/0002032, 5th ICTP School on Nonaccelerator Astroparticle Physics, Trieste, Italy, 29 Jun 10 Jul 1998; E. Aslanides et al. [ANTARES Collaboration]: astro-ph/9907432

28. J. Arafune, T. Yoshida, S. Nakamura and K. Ogure: hep-ph/0005103

29. M. Axenides, E. G. Floratos, G. K. Leontaris, N. D. Tracas: Phys. Lett. B 447, 67 (1999)

30. M. Colpi, S. L. Shapiro, S. A. Teukolsky: Astrophys. J. 414, 717 (1993) K. Sumiyoshi, S. Yamada, H. Suzuki, W. Hillebrandt: astro-ph/9707230 